\title{
11 from 11
}

\author{
Elvin Beach
}

Published online: 27 January 2022

(C) ASM International 2022

ASM International has recently released updated versions of the Handbooks focused on failure analysis and prevention. The Journal of Failure Analysis and Prevention (JFAP) has the privilege of offering a preview of some of the new and revised articles from the new Handbooks in the first issue of 2022. A brief introduction of the articles available in this issue is provided here.

ASM Handbook, Volume 11: Failure Analysis and Prevention, provides information on topics suitable for new professionals in the field all the way up to state-of-the-art discussions of specific tools, techniques, fracture modes, and failure types. The revised edition of Volume 11 (available both digitally and in print beginning in March 2021) begins with a thoughtfully composed article that familiarizes the topic to those new to the field. The revised article, "Introduction to Failure Analysis and Prevention," written by three of the Volume 11 volume editors (Shipley, Miller, and Parrington), defines categories of failures, summarizes root-cause analysis, and discusses failure prevention strategies. This article is useful both to new professionals and as a concise review for experienced professionals in the field. Another introductory article highlighted and published in this issue of JFAP is "The Failure Analysis Process - An Overview" by Aliya. This article walks readers through topics including "Principles and Approaches to Failure Analysis Work," the "Open Mind/Open Toolbox Approach," and the "Importance of

E. Beach, Editor-in-Chief, Journal of Failure Analysis and Prevention.

E. Beach $(\bowtie)$

Materials Science \& Engineering Department, 1044 Fontana Laboratory, The Ohio State University, 140 West 19th Avenue, Columbus, OH 43210, USA

e-mail: beach.110@osu.edu
Communication." These two introductory articles should be of interest to most readers as a review of solid fundamental practices.

There are several new articles in the updated Volume 11 Handbook that are included in this issue of JFAP as a sampling of the content. First, a set of articles on Nondestructive Evaluation (NDE) have been contributed to the Handbook. "Nondestructive Evaluation Applications for Failure Analysis" by Bartha presents an overview of inspection methods, characterizing crack sizes, and incorporating NDE results into the failure analysis process. An excellent complement to Bartha's article is included in the Tools and Techniques section of Volume 11. "Nondestructive Testing in Failure Analysis" by Lampman, Mulherin, and Shipley provides details on techniques including eddy-current inspection, radiography, and computed tomography. These articles summarize an important and growing field for both failure analysts and those working to prevent failures by inspection and quality control. Two more new articles in Volume 11 deal with the important field of compositional analysis. The article "Bulk and Microscale Composition Analysis" by Wright describes the "toolbox for bulk and microscale chemical analysis techniques in failure analysis of various types of materials." This is a well-written introduction that describes both established techniques and techniques that have been improved through new developments in technology such as real-time $\mathrm{x}$-ray mapping using silicon drift detector technology in energy dispersive $\mathrm{x}$-ray spectroscopy (EDS). There are new articles in the Tools and Techniques section that provide more in-depth information about chemical analysis techniques, which are good follow-on guides for practitioners. One of these articles is "Quantitative Chemical Analysis of Metals in Failure 
Analysis" by Akyuz and McKay. This article provides details on techniques such as inductively coupled plasma optical emission spectroscopy (ICP-OES), x-ray spectroscopic methods, and ion chromatography. The article is a nice addition to Volume 11 and provides details on the applicability and limitations of these techniques used in failure analyses.

The final $J F A P$-highlighted article from the updated Volume 11 is in the Wear Failures section of the Handbook. "Adhesive Wear Failures" by Podgornik is a welcome addition to the Handbook and provides details on the main forms of adhesive wear failures: seizing, galling, scoring, and scuffing. This 20-page article in the Handbook provides details on the underlying mechanisms and conditions necessary for adhesive wear failures to occur. Outstanding figures are found throughout the article, laboratory testing methods are reviewed, and nearly 180 references on the topic are provided in this exceptional addition to Volume 11.

A new edition to the ASM Handbook set, Volume 11A: Analysis and Prevention of Component and Equipment Failures, focuses on the useful service life, predicting the time to failure, and failure modes of engineering components. This Handbook was recently published both digitally and in print in December 2021. There are ten new articles in Volume 11A, four of which are highlighted and printed in this issue of JFAP. The first of the four articles is found in the Structural Life Assessment Methods section. The article "Assessment of Damage to Structures and Equipment Resulting from Explosion, Fire, and Heat Events" by Pierorazio, Cherolis, Lowak, Benac, and Edel walks the reader through damage indicators for each type of catastrophic failure listed in the title. The figures and photographs from failure investigation scenes are a valuable reference for new-to-the-field and seasoned analysts. Figure 8 for example shows how geographic information systems, pressure/explosion calculations, and understanding of the facility contribute to root cause determination during an investigation into such matters.

Volume 11A contains a section titled Failure Analysis of Metallic Components. An interesting new article in this section is "Failure Analysis of Medical Devices" by Bowers, Ganot, Malito, Kondori, Ezechukwu, Svedlund, and James, which is a comprehensive survey of medical implants that discusses failures of hip replacements, stents, and bone screws. This article introduces the challenging loading conditions and environments that can cause unexpected failures in metallic medical devices used in-vivo.

The last, but not least, of the articles from the new Handbook 11A included in this issue of JFAP are "Corrosion and Remaining Life Assessment" by Corleto and Hoerner and "Prevention of Machining-Related Failures" by Finn and Tartaglia. Both of these articles provide useful information to help assess parts in service or current processing methods to prevent any catastrophic failure from occurring. These articles are important additions to the Handbook and worth finding time to read.

The team at ASM and JFAP hope this summary and the chance to read some of the new and updated content in Handbooks 11 and 11A are useful. Both Handbooks are available in digital form and in print currently for those interested in reading all of the new articles and revisions in the current versions.

Publisher's Note Springer Nature remains neutral with regard to jurisdictional claims in published maps and institutional affiliations. 